\title{
The Clinical Analysis of the Nasal Septal Cartilage by Measurement Using Computed Tomography
}

\author{
So Min Hwang, \\ On Lim, \\ Min Kyu Hwang, \\ Min Wook Kim, \\ Jong Seo Lee \\ Aesthetic, Plastic and Reconstructive Surgery \\ Center, Good Moonhwa Hospital, Busan, \\ Korea
}

No potential conflict of interest relevant to this article was reported.

\begin{abstract}
Background: The nasal septal cartilage is often used as a donor graft in rhinoplasty operations but can vary widely in size across the patient population. As such, preoperative estimation of the cartilaginous area is important for patient counseling as well as operating planning. We aim to estimate septal cartilage area by using facial computed tomography (CT) studies.

Methods: The study was performed using facial CT images taken from 200 patients between January 2012 to July 2015 . Using the mid-sagittal image, the boundary of cartilaginous septum was delineated from soft tissue using the mean difference in signal intensity (or brightness). The area within this boundary was calculated. The calculated area for septal cartilage was then compared across age groups and sexes.

Results: Overall, the mean area of nasal septal cartilage was $8.18 \mathrm{~cm}^{2}$ with the maximum of $12.42 \mathrm{~cm}^{2}$ and the minimum of $4.89 \mathrm{~cm}^{2}$. The cartilage areas were measured to be larger in men than in women $(p<0.05)$. The area decreased with advancing age $(p<0.05)$.

Conclusion: Measuring the size of septal cartilage using brightness difference is more precise and reliable than previously reported methods. This method can be utilized as the standard for prevention of postoperative complication.
\end{abstract}

Keywords: Nasal cartilage / Computed tomography / Measurement

\section{INTRODUCTION}

The nasal septum is the first structure in the upper airway and consists of nasal septal cartilage and bony structures, including perpendicular plate of ethmoid and the vomer. Clinically, the septal cartilage is important as it is used as a donor cartilage for many rhinoplasty operations $[1,2]$. The amount of nasal septum occupied by the cartilaginous component is different across individual patients, and this leads to a wide variations in the shape and size of the nasal septal cartilage. Hence, it is important to assess the amount of

\section{Correspondence: So Min Hwang}

Aesthetic, Plastic and Reconstructive Surgery Center, Good Moonhwa Hospital, 119 Beomil-ro, Dong-gu, Busan 48735, Korea

E-mail: sominhwang@hanmail.net

Received May 25, 2016 / Revised September 12, 2016 / Accepted September 12, 2016 septal cartilage available for harvest. Nevertheless, no standard method of preoperative evaluation exists for septal cartilage harvest.

In previous studies, the dimensions of nasal septal cartilages were studied in cadavers, magnetic resonance imaging (MRI), and computed tomography (CT) [3-6]. Although the cadaveric measurements are accurate, the data is not relevant in the clinical setting [3,4]. MRI measurement is useful in investigating differences between nasal septal cartilage and soft tissue. However, the high cost of MRI prohibit widespread use for aesthetic rhinoplasty application, and thus it is very rare to have MRI data available in the relevant clinical setting [5]. On the other hand, CT is the most frequently used technique to identify the fracture and deviation of the nasal septum in the setting of facial injury, and is relatively inexpensive when compared to MRI, with the highest clini- 
cal accessibility [1]. In addition, the brightness can be compared between tissues only by a simple operation of the program, enabling more accurate assessment of the septal cartilage dimensions.

In this study, we measured brightness differences between the subcutaneous tissue and the cartilage objectively using a software solution. Based on the brightness data, we were able to delineate the boundaries of septal cartilage. This information can be important in preoperative planning for septal cartilage harvest, and can be used as reference data to prevent complications such as postoperative nose deformities.

\section{METHODS}

This study has been approved by Institutional Review Board of Inje University (No. 2015172-2) and followed its Code of Ethics for Scientific Research.

This retrospective study was carried out for patients who had visited our hospital and undergone CT scans for 3.5 years from January 2012 to July 2015. Patients less than 18 years of age were excluded from the study, as rhinoplasty is not performed in the pediatric and adolescent group. Also, patients were excluded for severe septal deviation or the presence of septal fracture.

CT images were obtained at $120 \mathrm{kV}$ and $180 \mathrm{~mA}$. For the measurement of the area, first the point was set where both cerebral hemispheres were divided. Subsequently, the sagittal images were extracted using the PiViewSTAR software (INFINITT software, Seoul, Korea). The boundary of the cartilage was marked on the extracted image before the area was measured.

We used the brightness difference between tissues when marking the boundary in order to ensure the objectivity in distinguishing the septal cartilage tissue from the subcutaneous tissue of the nose. This method is mainly used for non-destructive tests, and measures the brightness difference created between the different tissues, which could be confirmed using the existing program (Fig. 1). On each CT image, 3 points were selected excluding the sections in contact with the bones. From the points, the brightness difference was measured, after which the areas exhibiting drastic differences were marked (Fig. 2). With these as starting
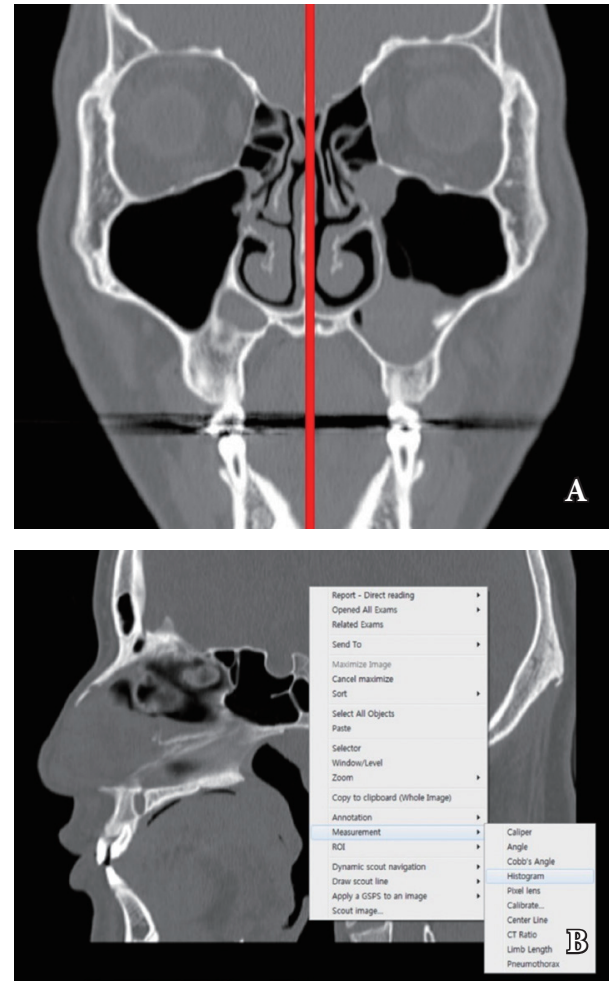

Fig. 1. (A) Setting of midline and measurement of septal cartilage area. The mid-sagittal plane was used as the reference plane. (B) The midsagittal computed tomography image. Identifying of the difference of brightness between cartilage tissue and subcutaneous tissue. The histogram of brightness can be used to differentiate between tissues.

points, the boundary of the area was marked.

Then, the boundary of septal cartilage was marked in the extracted image using the GoodNotes software (Time Base Technology, Quarry Bay, Hongkong), followed by the boundary of cartilage adjacent to the perpendicular plate of ethmoid and vomer. Subsequently, the area was measured using the Paint.net software (dotPDN, Olympia, WA, USA) (Fig. 3). We measured the area and brightness for all images in each study. All measurements were performed twice and averaged.

Statistical analysis was performed using MedCalc Statistical Software ver. 16.4.3 (MedCalc Software bvba, Ostend, Belgium; https://www.medcalc.org; 2016). Independent samples t-test and analysis of variance test were conducted to determine the differences. Differences were considered to be statistically significant when the $p$-value was less than 0.05 . 

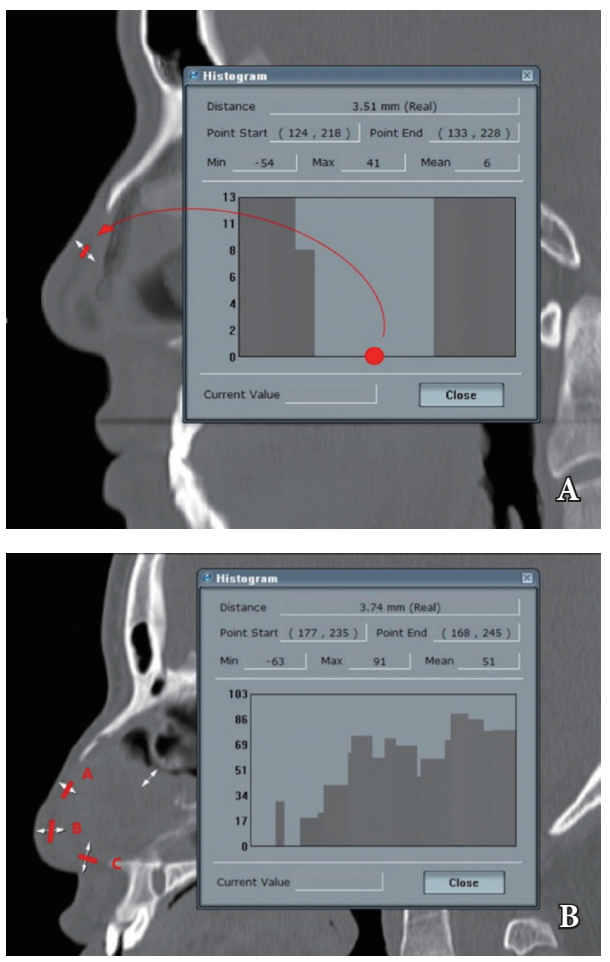

Fig. 2. (A) We set one point on the image that is between anteriormost nasal tip and inferior edge of nasal bone. From the points, the brightness difference between subcutaneous tissue and septal cartilage was measured. And we marked the margin of septal cartilage. (B) On computed tomography image, 3 points were selected excluding the sections in contact with the bones. A, Median point between $\mathrm{B}$ point and inferior edge of the nasal bone; B, Anteriormost end of the septal cartilage; $\mathrm{C}$, Inferiormost point of the suture line between the septal cartilage and perpendicular plate of ethmoid bone.

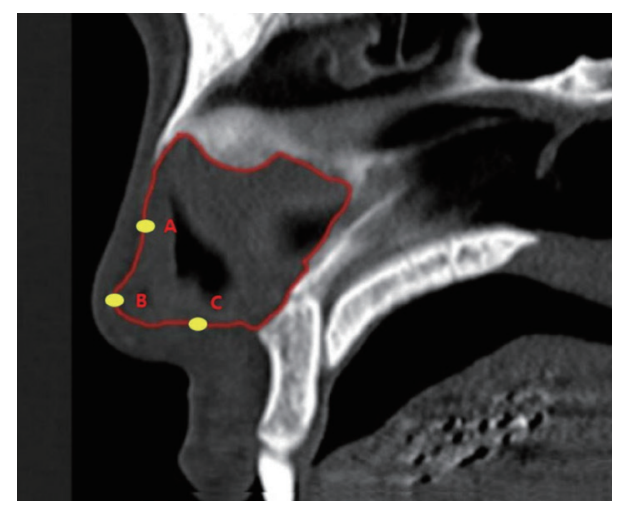

Fig. 3. Boundary of septal cartilage (red line).

\section{RESULTS}

The review identified 200 patients meeting inclusion criteria (136 males and 64 females). The mean age was 37.1 years. Facial injury was the most common indication for the facial CT. Other indications included nasal obstruction and preoperative evaluation for bimaxillary surgery.

The mean brightness of subcutaneous tissue was 3.83, ranging from -45 to 44 , with a standard deviation of 12.12 . The mean brightness of septal cartilage was 54.56, ranging from 4 to 75 , with a standard deviation of 10.87. Consequently, the mean difference between the two tissues was 50.73 ranging from 8 to 87 , with a standard deviation of 7.34 .

The mean area for septal cartilage was measured to be $8.18 \mathrm{~cm}^{2}$, ranging from 4.89 to $12.42 \mathrm{~cm}^{2}$, with a standard deviation of 1.38 . In males, the mean area for septal cartilage was $8.57 \mathrm{~cm}^{2}$ while it was $7.36 \mathrm{~cm}^{2}$ in females, and this difference was significant $(p<0.05)$ (Table 1). With respect to age, the mean area was measured to be $9.89 \mathrm{~cm}^{2}$ for the 18 -to-20 year old group, $8.68 \mathrm{~cm}^{2}$ for the 21-to-30 year old group, $8.23 \mathrm{~cm}^{2}$ for the 31 -to- 40 year old group, $7.79 \mathrm{~cm}^{2}$ for the 41 -to-50 year old group, and $7.02 \mathrm{~cm}^{2}$ for the 51 -to78 year old group. This decrease in septal area with increasing age was statistically significant $(p<0.05)$ (Table 2$)$.

\section{Case}

A 29-year-old woman presented with a flat nose and bulbous tip and

Table 1 . Septal cartilage area by sex

\begin{tabular}{lc} 
Sex & Septal cartilage $\left(\mathrm{cm}^{2}\right)$ \\
Male $(\mathrm{n}=136)$ & $8.57 \pm 1.35$ \\
\hline Female $(\mathrm{n}=64)$ & $7.36 \pm 1.03$ \\
\hline Total $(\mathrm{n}=200)$ & $8.18 \pm 1.38$ \\
\hline
\end{tabular}

Values are presented as average \pm standard deviation.

A $p$-value $<0.001$, independent samplest-test.

Table 2. Septal cartilage area by age

\begin{tabular}{lc} 
Age $(y r)$ & Septal cartilage $\left(\mathrm{cm}^{2}\right)$ \\
\hline $18-20(n=20)$ & $9.89 \pm 1.34$ \\
$21-30(n=60)$ & $8.68 \pm 1.29$ \\
\hline $31-40(n=43)$ & $8.23 \pm 1.05$ \\
$41-50(n=32)$ & $7.79 \pm 0.79$ \\
$>51(n=45)$ & $7.02 \pm 0.94$ \\
\hline
\end{tabular}

Values are presented as averagetstandard deviation.

A $p$-value $<0.001$, analysis of variance test. 


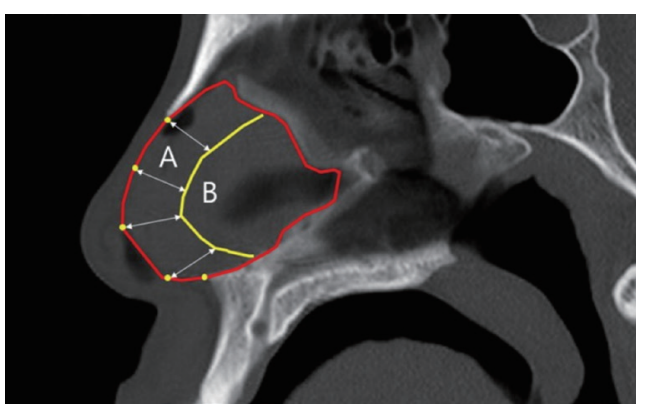

Fig. 4. Computed tomography image from the presented case. We were able to measure the harvestable amount of nasal septal cartilage. $A+B$, total septal cartilage; $\mathrm{B}$, harvestable nasal septal cartilage. Doublearrow lines represent $1-\mathrm{cm}$ offset as the L-strut width.

wished to undergo cosmetic surgery. An augmentation rhinoplasty was planned, with tip plasty and septal extension graft. Because septal cartilage was going to be used as a donor graft, a facial CT was obtained to assess the amount of cartilage available for septal graft (Fig. 4). Using the methods described above, the area of the nasal septal cartilage was measured to be $7.45 \mathrm{~cm}^{2}$. To preserve the L-strut, the nasal septal cartilage was designed by referencing the CT image. The area measurement excluding the L-strut was estimated to be 4.78 $\mathrm{cm}^{2}$. This was deemed be a sufficient amount of cartilage. Then the operator read the location of the cartilage, the operation was performed without any difficulty.

\section{DISCUSSION}

In the context of rhinoplasty operations, nasal septal cartilage is often used as a structural scaffold within the same operative site as the operation itself [7-9]. Among cartilage graft options for rhinoplasty, the costal cartilage is the most distant from the operative site and leaves a scar after surgery. The auricular cartilage has low rigidity compared to the septal cartilage. Hence, the septal cartilage is preferred when the cartilage is to be collected for the structural scaffold $[9,10]$.

In addition to the direct cartilage transplantation, an indirect method is also favored, in which chondrocytes that have been already collected are injected into a polyglycol framework and cultured therein, followed by grafting into the area for reconstruction. However, the cartilage is mainly composed of collagen and proteoglycan, and the number of chondrocytes is low in adults [7]. As such, chondrocytes are preferably collected from the front and middle sections of the nasal septal cartilage, where the chondrocytes are most abundant and have the highest viability [11].

The septal cartilage is an important structure not only for direct transplantation but also for indirect transplantation using tissue engineering in reconstructive surgery. Hence, preoperative evaluation is considered essential in the operations requiring septal cartilage grafts. According to previous studies, the area of nasal septal cartilage was measured to be $8.4 \mathrm{~cm}^{2}$ for males and $7.9 \mathrm{~cm}^{2}$ for females in cadavers [3], $7.5 \mathrm{~cm}^{2}$ on CT scans [7], and $8.9 \mathrm{~cm}^{2}$ on MRI [5]. In general, the nasal septal cartilage increases rapidly in size from birth through adolescence. After the adolescent phase, some of the cartilage is gradually ossified in the aging process and thus the area of the septal cartilage decreases in relation to the whole nasal septum [1]. So far, studies on septal cartilage sizes between men and women have offered contradictory results, with some suggesting significant difference while others do not [5].

The recent development in nasal tip plasty using cartilage graft has increased the interest in septal cartilage. However, it has been difficult to estimate the anatomic features of septal cartilage prior to the actual operation. In the past, CT had low accessibility and was unable to resolve cartilaginous areas due to the low resolution and the problem of radiation dose, all of which limited the use of CT for rhinoplasty operations. Consequently, MRI was a main tool for such purpose [12]. However, the recent progress in CT technology has lead to differentiation of the subcutaneous fat layer in the nasal region, and it has become easier to confirm boundary for septal cartilage. At the same time, clinical accessibility of CT has been greatly improved [7]. In addition, facial CT has become a common place preoperative workup for nasal fracture, for which fracture reduction is often followed by cosmetic operation.

In a study measuring the area of septal cartilage using facial CT, Kim et al. [6] marked and connected 7 specific points on the boundary of the septal cartilage and estimated the cartilaginous area to be $7.5 \mathrm{~cm}^{2}$. In a subsequent study by the same group, the area of the septal cartilage was more finely measured by marking the boundary itself of the septal cartilage. The mean area was found to be $8.3 \mathrm{~cm}^{2}$ with the measured areas ranging from a minimum of 4.4 
$\mathrm{cm}^{2}$ to a maximum of $14.1 \mathrm{~cm}^{2}$, thus showing the large differences in the septal cartilage area across the sample population [1]. Therefore, we utilized brightness difference between tissues to more accurately measure the area of cartilaginous septum.

Histologic composition of the septal cartilage is $77.7 \%$ water, $7.7 \%$ collagen and $2.9 \%$ sulfated glycosaminoglycans [7]. The cartilage must show difference in brightness when compared with subcutaneous tissues due to the difference in this histologic composition, based on which we have devised the method of assessing septal cartilage.

We have found that the mean brightness of the subcutaneous tissue was 3.83 with the minimum of -45 and the maximum of 44, showing significant differences across individuals. The mean brightness of the cartilage was 50.73 with the minimum of -4 and the maximum of 75 , also showing individual differences. Although there was an overlap of the intensities between the two tissues, the mean difference in the brightness between the subcutaneous tissue and the cartilage in an individual was 50.73 with the minimum of 8 and the maximum of 87 , which was clear enough to distinguish between the two tissues without equivocation.

Using this data, the septal cartilage areas were compared according to age and sex. The result indicated that the nasal septal cartilage was larger in men than in women and that it began to decrease in size after the second decade of life. The post-adolescent reduction in septal cartilage size is due to the ossification process during which septal cartilage transitions into perpendicular plate of ethmoid bone, a conclusion supported by our study.

Accurate assessment of cartilaginous area is helpful because it assist in preoperative assessment for rhinoplasty patients. If the CT-measured cartilage is inadequate for the operation planned, then the operator knows to inform the patient that a secondary donor site is needed for cartilage graft harvest. Another benefit in preoperative assessment of septal cartilage is that it allows the surgeon to estimate how much graft can be taken while preserving an adequate L-strut behind.

The use of brightness information to delineate septal cartilage and to calculate its size is more elaborate and may be more accurate, when compared to methods introduced in previous studies. It is possible that the values obtained using our method are more reliable than the previous measurements $[1,5]$.

There are several limitations in our study which may have influenced the results. There can exist various biases including overlapping between the septal cartilage and alar cartilage or the overlapping of keystone areas and also anatomical variations. To minimize the influence these factors have on the outcomes, the authors measured the distance between the CT scan slice as 0.75 $\mathrm{mm}$, which is about $1 / 3$ of the usual measured value. The purpose of this was to minimize that amount of overlapping that occurs between the structures.

Even if the method in our study is accurate, it was impossible to measure all the brightness differences at the entire boundary between the subcutaneous tissues and cartilage tissue. This problem can be overcome, if a program that can recognize the brightness difference between the two tissues and set the boundary accurately. In the future, it will be possible to measure the area of the nasal septal cartilage accurately before surgery. Such a progress will provide sufficient information and data to not only plastic surgeons but also surgeons in other areas such as otolaryngology, eventually leading to accurate operation and good outcomes.

CT studies can be very useful in the preoperative evaluation of the nasal septal cartilage. Using the brightness difference, we were able to define a more accurate boundary for the cartilaginous septum. CT studies can be very useful in the preoperative evaluation of the nasal septal cartilage. Using the brightness difference, we were able to define a more accurate boundary for the cartilaginous septum. In order to be this method more automated and objective, there is need for the additional technology that can automatically recognize and indicate the boundary of the septal cartilage. So the measurement of the nasal septal cartilage by using CT is more widespread in the future, such a method can provide crucial data for surgery using the septal cartilage and also for the prevention of complications such as postoperative nose deformities.

\section{REFERENCES}

1. Kim JH, Jung DJ, Kim HS, Kim CH, Kim TY. Analysis of the development of the nasal septum and measurement of the harvestable septal cartilage in Koreans using three-dimensional facial bone computed 
tomography scanning. Arch Plast Surg 2014;41:163-70.

2. Sajjadian A, Rubinstein R, Naghshineh N. Current status of grafts and implants in rhinoplasty: part I. autologous grafts. Plast Reconstr Surg 2010;125:40e-49e.

3. Miles BA, Petrisor D, Kao H, Finn RA, Throckmorton GS. Anatomical variation of the nasal septum: analysis of 57 cadaver specimens. Otolaryngol Head Neck Surg 2007;136:362-8.

4. Kim JS, Jang PY, Choi TH, Kim NG, Lee KS. The dimension of the septal cartilage using the cadaver study. J Korean Soc Aesthetic Plast Surg 2006;12:29-32.

5. Kim IS, Lee MY, Lee KI, Kim HY, Chung YJ. Analysis of the development of the nasal septum according to age and gender using MRI. Clin Exp Otorhinolaryngol 2008;1:29-34.

6. Kim J, Cho JH, Kim SW, Kim BG, Lee DC, Kim SW. Anatomical variation of the nasal septum: Correlation among septal components. Clin Anat 2010;23:945-9.

7. Homicz MR, McGowan KB, Lottman LM, Beh G, Sah RL, Watson D.
A compositional analysis of human nasal septal cartilage. Arch Facial Plast Surg 2003;5:53-8.

8. Collawn SS, Fix RJ, Moore JR, Vasconez LO. Nasal cartilage grafts: more than a decade of experience. Plast Reconstr Surg 1997;100:154752 .

9. Tardy ME Jr, Denneny J 3rd, Fritsch MH. The versatile cartilage autograft in reconstruction of the nose and face. Laryngoscope 1985;95:523-33.

10. Gunter JP, Rohrich RJ. External approach for secondary rhinoplasty. Plast Reconstr Surg 1987;80:161-74.

11. Vetter U, Heit W, Helbing G, Heinze E, Pirsig W. Growth of the human septal cartilage: cell density and colony formation of septal chondrocytes. Laryngoscope 1984;94:1226-9.

12. Kim JE, Park HS, Yoon CH, Kim HJ. Analysis of nasal septal fracture combined in nasal bone fracture using computerized tomography. J Korean Soc Plast Reconstr Surg 1998;25:852-9. 